\title{
A SURVEY AND REVIEW OF GSM BASE TRANSCEIVER SYSTEM INSTALLATION, ARCHITECTURE AND UPLINK/DOWNLINK
}

\author{
Manjish Adhikari ${ }^{1 *}$ \\ ${ }^{* 1}$ B. Tech Senior year, Department of Electronics and Communications Engg. Jawaharlal Nehru Technological \\ University, Kakinada, A.P, India adhikari.manjish@gmail.com
}

*Corresponding Author: -

Email: adhikari.manjish@gmail.com

\begin{abstract}
: -
With the advent of development in LTE system, eNodeB the revolutionised version of BTS is used for a connection between BSC and the users. A better and improved system increases the capacity and the functionality of the mobile wireless communication system. A Base Transceiver System (BTS) is a system in a mobile communication network that houses radio receivers and is used for wireless communication between users and network providers that is under the control of Base Switching Controller (BSC) and then the exchange. This paper deals with the study of a ground-based GSM Base Transceiver System (BTS) and its installation process, architecture, internal structures, the process involved in the uplink and downlink call procedures and the future works. This study is based on the visit to a ground based BTS at BSNL regional training centre (RTTC), Hyderabad during the EETP course. The observations made are duly recorded, reviewed and presented for a better understanding of a mobile BTS system.
\end{abstract}

Keywords: BTS, BSC, GSM, LTE, Prefab shelter, Baseband signal, Uplink, Downlink,

\section{(c) $(\$)$}




\section{OVERVIEW}

A mobile network consists of Mobile Station (MS), Base Station Subsystem (BSS), Network Switching Subsystem (NSS) and Operator Support Subsystem (OSS). The Base Station subsystem is divided into two parts- BSC and BTC. A BTS, also referred to as the radio base station (RBS), node B (in 3G Networks) or, simply, the base station (BS) or evolved node eNB in LTE standard is a system that has the radio transceivers which define a cell and provides wireless communication between users

like mobile phone, computers or WLL phones and a network service provider. A BTS is controlled by a Base Station Controller (BSC). A BTS is usually placed in the centre of a cell whose transmitting power defines the size of a cell. Each BTS has between 1 to 16 transceivers, depending on the density of users in the cell. Each BTS serves as a single cell. A BTS has four main parts namely power element, a power source (engine/alternator), a BTS machine and Towers \& antenna.

Usually while installing the BTS certain parameters are to be considered and certain standard procedures are to be followed.

\section{SITE SELECTION}

Ground based BTS system should be kept at a place where RF survey is performed first. There shouldn't be any high tension lines around that could interfere with the signals at the BTS. The area should have an easy access with the transport facility so that operation and maintenance can be easily done. The area where the BTS is to be installed should be levelled and with a power source nearby. Power source, engine alternator, pre fab shelter and tower foundation site should be selected properly.

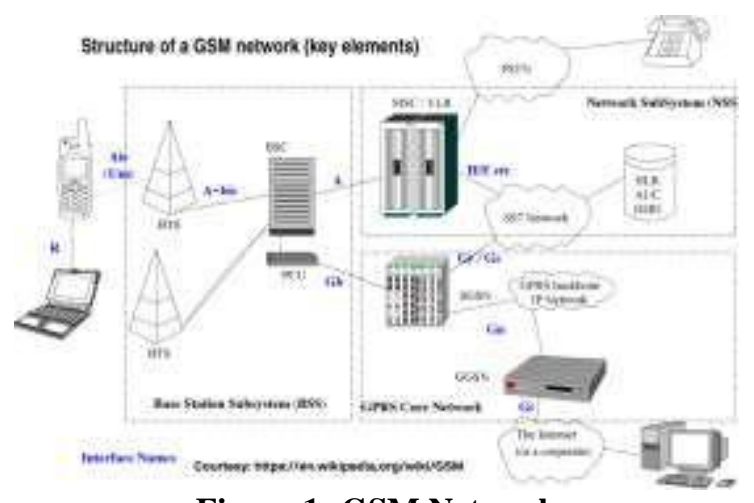

Figure 1- GSM Network

\section{BTS/ TELECOM SHELTER}

A BTS is a closed chamber which should be sealed from the external environment and away from the heat, weather and noise. The shelter should be light weight and easy to maintain with the four walls, root, floor and the door, (perfect sealing). Usually having a dimension of $4000 \mathrm{~L} * 3500 \mathrm{~W} * 3000 \mathrm{H}$ (all in $\mathrm{mm}$ ). The temperature within the shelter should be maintained at $25+/-2^{\prime} \mathrm{C}$

\section{Technical Specifications of a Pre fab Shelter under study}

I) Roof structure and cover-pre-painted galvanized sheets of $0.4 \mathrm{~mm}$ thick trapezoidal shelter laid over a framework of truces, columns and purling.

II) Wall - Providing and fixing of walls using 50/75 mm thick interlocking aerated concrete wall panels made of two 5 mm thick cement fiber boards conforming to IS 14276- 1995.

III) False ceiling- Using 595*595*12 mm mineral fiber board/gypsum. Placed on suspended grid frame to form $600 * 600$ mm grid suspended ceiling.

IV)Doors- made of pressed steel using powder coated CR coil $1.25 \mathrm{~mm}$ thick, shutter of $32 \mathrm{~mm}$ thick ERP flush door.

V) Windows- made of pressed steel using powder coated CR $1.25 \mathrm{~mm}$ Thick C channels of size $30 * 52 \mathrm{or} 77 \mathrm{~mm}$.

VI) Painting- 2 coats of acrylic paint outside and 2 coats of oil bound distemper inside. (Source: BSNL EETP India)

A BTS is usually associated with GSM or CDMA and is able to encrypt and decrypt communication signals, spectrum filter and wirelessly communicate.

\section{IV.ARCHITECHTURE OF BTS}

The general architecture of BTS system reveals the following parts.

A. Power Cards: A power card is used to provide fixed current and voltage levels to circuit components. A BTS usually uses $-48 \mathrm{~V}$ power whose positive is grounded to reduce noise. 


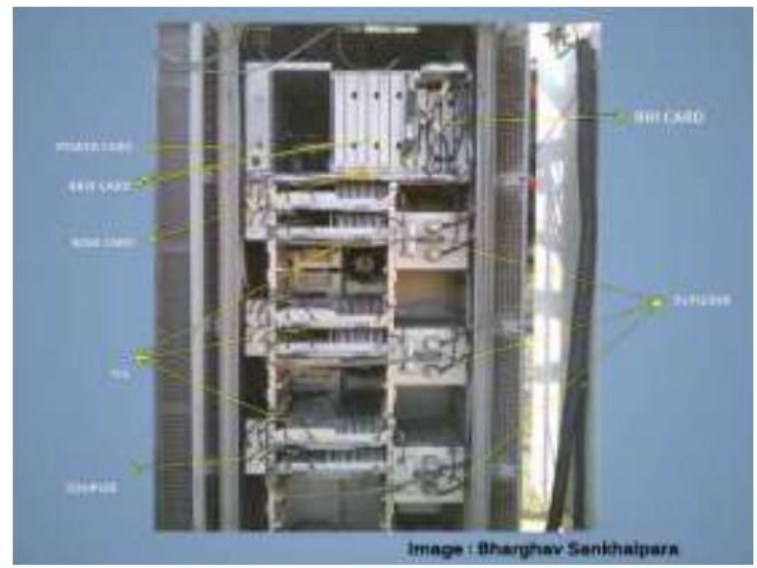

Figure 2- Architecture of BTS

B. Baseband receiver Unit (BB2F): A BB2F is used for digital signal processing and frequency hopping. It connects the BOIA card to TRx.

C. Transceiver (TRx)/ Data Receiver (DRx): Transceivers handles the user calls. Usually there are 12 TRx in a BTS and can handle 8 calls/sec.

D. Base Operation and Interface Unit (BOIA)- It processes the baseband signals received from the BB2F and interfaces the processed signal with transmission cards such as RRI, E1/T1 etc. Also BTS initialisation, power amplification, O\&M signalling, clock functions, timing functions, etc. So it is considered as the brain of the BTS.

E. Radio Receiver Card(RRI) : It provides E1 connectivity to the BTS. Also it creates the microwave link between BTS \& BSC.

F. Multicouplers and Duplexers: Multicouplers are used to connect different TRxs also into the duplexer.

G. Alarm Extension System : It monitors and collects the working state of various units of BTS and then extends to the O\&M monitoring station.

\section{TOWERS AND ANTENNAS:}

Usually the tower is ground based or roof top based; roof top based used in the congested city area. GBT are near the BTS having height of usually $30-40 \mathrm{~m}$. Towers may be self-supporting or guyed tower. Based on their applications towers may be of following types-

A. Microwave Towers: They are used for long distance communication and has 30-100 m height.

B. Triangular Tubular Hybrid Towers(TTHT): BSNL uses them in rural areas of 40,60,80 or 100m heights. Usually it contains $15 \mathrm{~m}$ mast; 5 panels each of $3 \mathrm{~m}$ height.

C. For GSM technology $40 \mathrm{~m}$ high towers are used. The steel tower material has legs of M.S. angles of grade A as per IS:

2062-1999 \& IS: 808-1989, materials for nut and bolt are of grade 4.6; The fabrication of tower was done in accordance with IS 800-1984.

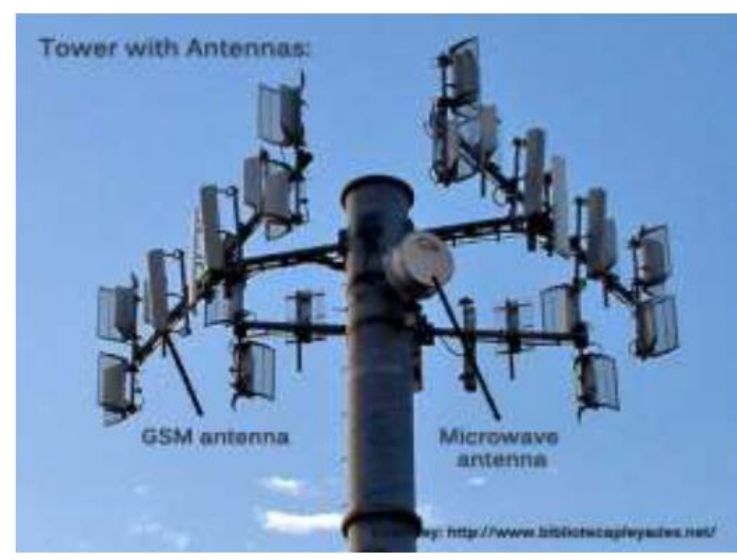

Figure 3. Antennas in BTS

Similarly, two major types of antennas are used in the BTS. They are:

A. GSM Antenna: It is used for the transmission and reception of the user's signals. It is plane or a dipole antenna.

B. Microwave/Drum Antenna: These are parabolic or horn antenna. It works based on Line-of-Sight propagation (LOS) and connects BTS to BTS or with the BSC. 
Antennas are usually directional so as to avoid power loss and more area coverage. To improve the quality of the signal usually a process called antenna diversity or space diversity is employed.

\section{VI.CALL PROCESS THROUGH BTS}

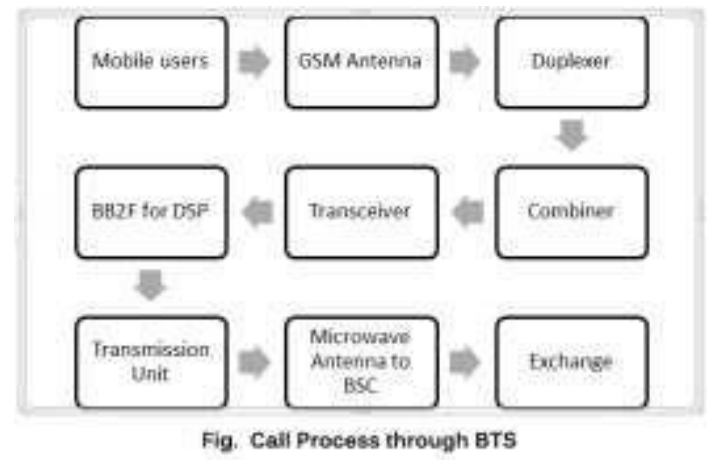

Usually, the path followed for the call process in uplink and downlink can be explained as the flow chart. The information sent by the mobile users are caught by the GSM antenna and via a duplexer into the transceiver which send the information to be processed by the BB2F. This processed information through the transmission unit like antenna is sent to the BSC which monitors it in the exchange. In the downlink process, the data stored in the internet or from other sources are sent to the users via similar path.

The customers within a cell are connected to a particular BTS and hence based on the power and capacity of BTS, customer number is determined. The BTS includes amplifiers that supply the appropriate broadcast RF power levels as well as boost the incoming RF signal power so that it is suitable for transmission to the BSC. Another function of the BTS is to convert between the appropriate protocols for broadcast to the wireless portion of the network or to convert the protocols for transmission to the BSC.

\section{CONCLUSION}

A BTS system is an important part of the mobile and wireless communication system that monitors the customers within the cell. Based on this system the capacity of network is determined so an effective BTS can result in high network capacity and better functionality.

\section{VIII.FUTURE WORKS}

With the advent of development in LTE system, eNodeB the revolutionised version of BTS is highly efficient. There are a lot of research opportunities in this area of LTE which is dedicated to providing faster data rate and better connectivity.

\section{IX.ACKNOWLEDGEMENT}

This study and review is mostly based on the findings of the visit and training at BSNL RTTC Hyderabad. I would like send my sincere gratitude to all teaching staffs of RTTC who guided us in learning the core backbone of mobile communication system and Department of ECE, JNTU Kakinada for providing the assessment opportunity.

\section{REFERENCES:}

[1]. Satoshi Maruyama, Katsuhiko Tanahasi, Takehiko Higuchi, Base Transceiver System for W-CDMA System

[2].Prefab Shelters and Towers, learntelecom.bsnl.co.in/EETP/BSNL_SI LVER_CERTIFICATION_COURSE/VER.0 2/ June 2014

[3].Peng Chenguyan http://www.dcs.gla.ac.uk/ lewis/teac hing/Tik-111.html

[4]. Base Transceiver Station https://en.wikipedia.org/wiki/base_tr ansceiver_system

[5]. Fibre Optic TransmitterinBase Station Application, Avago Technologies

[6]. L.A. Akinyemi, N.T.Makanjuola \& O. Shoewu, F.O. Edeko; African Journal of Computing and ICT Vol. 7 No.2 June 2014 Comparative Analysis Of Base Transceiver Station(BTS) and Power TransmissionLinesEffects OnThe Human Body Inthe LagosEnvirons, Lagos State, Nigeria.

[7]. Bhargav Shankhalpara; BTS Site Visit http://www.slideshare.net/bhargav_s hankhalpara/bts-visit

[8]. NasirFaruk, Mujahid Y. Muhammad, Olayiwola W. Bello, Abubakar Abdulkarim, Agbakoba John and Mohammed I. Gumel ; Energy Conservation through Site Optimization for Mobile Cellular Systems (Base Transceivers Station Optimization)

[9]. GSM Architecture "www.tutorialsports.com/gsm

[10]. YasirMalik, Kishwer Abdul Khaliq, Bessam Abdulrazak, Usman Tariq; MobileNodeLocalizationsinLocal Network

[11]. GNURadio: http://www.gnu.org/software/gnurad io

[12]. Marco Anisetti, Claudio A Ardagna, Valerio Bellandi, Ernesto Damiani, and Salvatore Reale. Advanced localization of mobile terminal in cellular network. International Journal of Communications, Network and System Sciences.1(1):95-103, 2008. 